\title{
Translating Endocannabinoid Biology into Clinical Practice: Cannabidiol for Stroke Prevention
}

\author{
Eugene L. Scharf ${ }^{*}$
}

\begin{abstract}
Introduction: The endocannabinoid system (ECS) regulates functions throughout human physiology, including neuropsychiatric, cardiovascular, autonomic, metabolic, and inflammatory states. The complex cellular interactions regulated by the ECS suggest a potential for vascular disease and stroke prevention by augmenting central nervous and immune cell endocannabinoid signaling.

Discussion: The endocannabinoid $\mathrm{N}$-arachidonoylethanolamine (anandamide) plays a central role in augmenting these processes in cerebrovascular and neurometabolic disease. Furthermore, cannabidiol (CBD), a nonpsychoactive constituent of Cannabis, is an immediate therapeutic candidate both for potentiating endocannabinoid signaling and for acting at multiple pharmacological targets.

Conclusion: This speculative synthesis explores the current state of knowledge of the ECS and suggests CBD as a therapeutic candidate for stroke prevention by exerting favorable augmentation of the homeostatic effects of the ECS and, in turn, improving the metabolic syndrome, while simultaneously stalling the development of atherosclerosis.
\end{abstract}

Keywords: cannabidiol; stroke prevention; obesity; vascular disease; anandamide

\section{Introduction}

The study of cannabis and endocannabinoid biology has recently been identified as a priority by the National Academy of Medicine. ${ }^{1}$ The extensive history of this medicinal plant has propelled the discovery of the human endocannabinoid system (ECS) and has opened a new therapeutic path for a multitude of disease states. Preclinical and human research has revealed the ECS to be pleiotropic and context-dependent regulator of stressed physiological states. Relevant to cerebrovascular neurology, the ECS has been shown to drive food intake and inhibit vascular inflammatory processes. This medical hypothesis suggests cannabidiol (CBD) as a therapeutic candidate for stroke prevention through its capacity to selectively augment and inhibit separate aspects of endocannabinoid biology simultaneously.

\section{Endocannabinoid Physiological Effects}

Endocannabinoids are arachidonic acid derivatives with widespread functions throughout physiology present in the central nervous system and circulation. The relevance of endocannabinoid function for stroke prevention will focus on two aspects known to be regulated by endocannabinoid function, neurometabolic balance, and vascular inflammation.

\section{Neurometabolic balance}

Observational evidence of the appetite-stimulating effects of herbal cannabis combined with knowledge of the pharmacological mechanisms of THC eventually identified dorsomedial, ventromedial, and lateral hypothalamic type 1 cannabinoid (CB1) receptors as a driver of reward-seeking behaviors, particularly food intake, reviewed by Cota et al. ${ }^{2} \mathrm{CB} 1$ receptors are described in the hypothalamus ${ }^{3}$ and endocannabinoids have high affinity for $\mathrm{CB} 1$ receptors. ${ }^{4}$ Administration of anandamide has been shown to stimulate appetite in mice. ${ }^{5}$ Finally, CB1 inverse agonist has been shown to be a potent appetite suppressant in clinical trials and improve several cardiometabolic health measures. 
Peripheral immune cell regulation

Two of the well-known endocannabinoids, anandamide and 2-arahidonoyl glycerol (2-AG), have different cellular responses at type 2 cannabinoid receptors. 2-AG increases migration in macrophage differentiated HL-60 cells, ${ }^{6}$ human natural killer cells, ${ }^{7}$ human B cells, ${ }^{8}$ and human eosinophilic leukemic cells but not neutrophils. ${ }^{9}$ Furthermore, 2-AG activates human umbilical vein endothelial cells promoting leukocyte migration by both $\mathrm{CB} 1$ and CB2 mechanisms. ${ }^{10}$ Mutations in $A B H D 12$, a gene that synthesizes the 2-AG degrading enzyme, have been linked to polyneuropathy, hearing loss, ataxia, retinitis pigmentosa, and cataract. ${ }^{11}$

In contrast, anandamide exerts anti-inflammatory effects by decreasing tumor necrosis factor-alpha (TNF$\alpha$ ) and interferon- $\gamma$ expression in human $\mathrm{T}$ cell cultures ${ }^{12}$ and decreasing expression of interleukin (IL)-6, IL-12, and interferon- $\gamma$ in prestimulated human dendritic cell cultures. ${ }^{13} \mathrm{CB} 2$ receptor agonists have also shown antiinflammatory effects in cell culture models involving human astrocytes prestimulated with IL- $1 \beta,{ }^{14}$ human endothelium prestimulated with TNF- $\alpha,{ }^{15}$ and human T-lymphocytes ${ }^{16}$ and neutrophils.

\section{Endocannabinoid Response to Disease}

Endocannabinoid receptors and their endogenous ligands are found to be altered in cardiovascular disease, hypertension, obesity, and diabetes. In congestive heart failure, human myocardial CB2 receptors are upregulated and CB1 receptors are downregulated, ${ }^{17}$ which has been attributed to inverse expression of the suppressive miRNA regulation of $\mathrm{CB} 1$ and $\mathrm{CB} 2$ receptor transcription. ${ }^{18}$ Elevated circulating levels of anandamide and cardiomyocyte $\mathrm{CB} 2$ receptor upregulation were present in both patients with aortic valve stenosis ${ }^{19}$ and congestive heart failure. ${ }^{17}$ Circulating levels of anandamide were significantly more elevated in humans with obstructive sleep apnea and diabetes when compared with controls and anandamide was also positively associated with increase in blood pressure. ${ }^{20}$

Plasma endocannabinoid levels were positively correlated with elevations in blood pressure when depressed women were compared with nondepressed controls $^{21}$ and elevated in diabetic men ${ }^{19}$ and obese postmenopausal women ${ }^{22}$ and obese adults. ${ }^{23} \mathrm{Circu}-$ lating 2-AG but not anandamide was significantly increased in visceral obese humans and positively correlated with visceral fat area, and circulating 2-AG was inversely associated with insulin sensitivity, even when controlling for body fat percentage. Abdominal fat mass was associated with alterations in circulating endocannabinoids. ${ }^{24}$ It is more likely that elevations in circulating endocannabinoids and altered CB1 and CB2 receptor expression are homeostatic reactions to the stresses of cardiac and metabolic syndrome disease states than primary causes of these disorders.

Both CB2 receptor agonists and antagonists have been reported to induce anti-inflammatory immune cell responses, the majority view is that peripheral immune CB2 receptor activation is anti-inflammatory. Furthermore, despite CBD having no pharmacological activity at the CB2 receptor, low doses of CBD appear similar to what is described with CB2 receptor activation in vitro and in vivo murine models of inflammation and atherosclerosis. Low dose THC arrests the development of a murine model of atherosclerosis through a CB2 receptor action. ${ }^{25}$ Intraperitoneal coadministration of both proinflammatory lipopolysaccharide (LPS) and 2-AG nullified the TNF- $\alpha$ serum response normally provoked by LPS alone in a murine model of inflammation. ${ }^{26}$

However, in murine experimental models of inflammatory pain, oral CBD doses as high as $20 \mathrm{mg} / \mathrm{kg}$ were not found to independently alter levels of TNF- $\alpha$ when compared with control. ${ }^{27}$ Anandamide and 2-AG inhibit the CD14 and TLR4 proinflammatory pathway, and synthetic CB2 agonists also show this effect as well. $^{28}$ In vitro TNF- $\alpha$ stimulated human coronary arterial endothelial cell activation and monocyte adhesion was blocked by a CB2 receptor agonist. ${ }^{15}$ Human macrophages laden with oxidized low-density lipoprotein (oxLDL or "foam cells") are believed to promote the development of atherogenesis through a subendothelial proinflammatory process.

In vitro data obtained with human cell lines support a role for peripheral circulating macrophage CB2 receptor activation as an anti-inflammatory and antiantherogenic mechanism. ${ }^{29}$ Human macrophages treated with a synthetic CB2 receptor agonist (JWH-015) were exposed to oxLDL in culture and were compared with controls. The central finding among the CB2activated cell group was reduced levels of intracellular oxLDL, proinflammatory cytokine TNF- $\alpha$, and IL- 12 . Interestingly, levels of 2-AG and anandamide were elevated in "foam cells" compared with controls. Is anandamide driving the formation of foam cells or responding to it? $\mathrm{CB} 2$ receptor agonist JWH-133 was shown to arrest TNF- $\alpha$ activation of human neutrophils in vitro by inhibiting phosphorylation of ERK1/ 2 . The level of neutrophil collagen degrading enzyme 
matrix metalloproteinase-9 (MMP-9) is increased in areas of low $\mathrm{CB} 2$ receptor expression, marker of plaque instability.

In humans, $\mathrm{CB} 2$ receptor expression is decreased in symptomatic carotid plaques as compared with asymptomatic plaques. ${ }^{30}$ Interestingly, it was later shown that CB1 receptor expression was undetected in human carotid plaque samples. ${ }^{30}$ Thus, preclinical models of atherosclerosis offer a possible role for endocannabinoid signal modulation as a method for stalling the development of atherosclerosis through reduction of the inflammatory drivers of this process. To the authors' knowledge, the effects of ischemic and hemorrhagic stroke on circulating endocannabinoid levels have not been investigated to date.

\section{Rationale for CBD in Cerebrovascular Disease Prevention \\ Cannabidiol}

The scientific literature has described $>65$ molecular targets for the nonpsychoactive phytocannabinoid CBD, including receptors, ion channels, and enyzmes, and transporters. These molecular targets make CBD relevant for a host of neurological diseases; for a review on the molecular targets of CBD, see Bih et al. ${ }^{31}$ Despite being named a cannabinoid, CBD has very low affin- ity for both $\mathrm{CB} 1$ (Ki: $1510 \mathrm{nM}$ ) and $\mathrm{CB} 2$ (CB2 Ki: $3612 \mathrm{nM}$ ) receptors and probably exerts no direct effect at CB2. Furthermore, CBD does not appear to exert a direct antagonist effect on CB1 receptors in pharmacological assays, yet CBD clearly shows "functional" antagonism of $\mathrm{CB} 1$ agonists, possibly through negative allosteric modulation. ${ }^{32,33} \mathrm{CBD}$ increases circulating endocannabinoids through inhibition of fatty acid amide hydrolase (FAAH) ${ }^{34}$ and competition for intracellular lipid catabolism. ${ }^{35,36} \mathrm{CBD}$ potentiates anandamide by reuptake inhibition of the anandamide membrane transporter (AMT) in vitro although this finding is controversial and needs to be replicated in vivo. ${ }^{37} \mathrm{CBD}$ has demonstrated activation of peroxisome proliferatoractivated receptor gamma (PPAR $\gamma),{ }^{38}$ adenosine $\mathrm{A} 2 \mathrm{~A},{ }^{39}$ and 5-HT1a serotonin receptors. ${ }^{40} \mathrm{CBD}$ is subject to first pass hepatic metabolism and is a potent CYP inhibitor, including clopidogrel prodrug metabolizing enzyme CYP2C19. ${ }^{31}$

\section{Obesity}

CBD is very likely a negative allosteric modulator of activated hypothalamic CB1 receptors exerting a homeostatic effect and reducing the strong hunger drive and improving weight loss. ${ }^{32}$ CBD has shown to attenuate $\mathrm{CB} 1$ receptor agonist-driven food intake in a rat
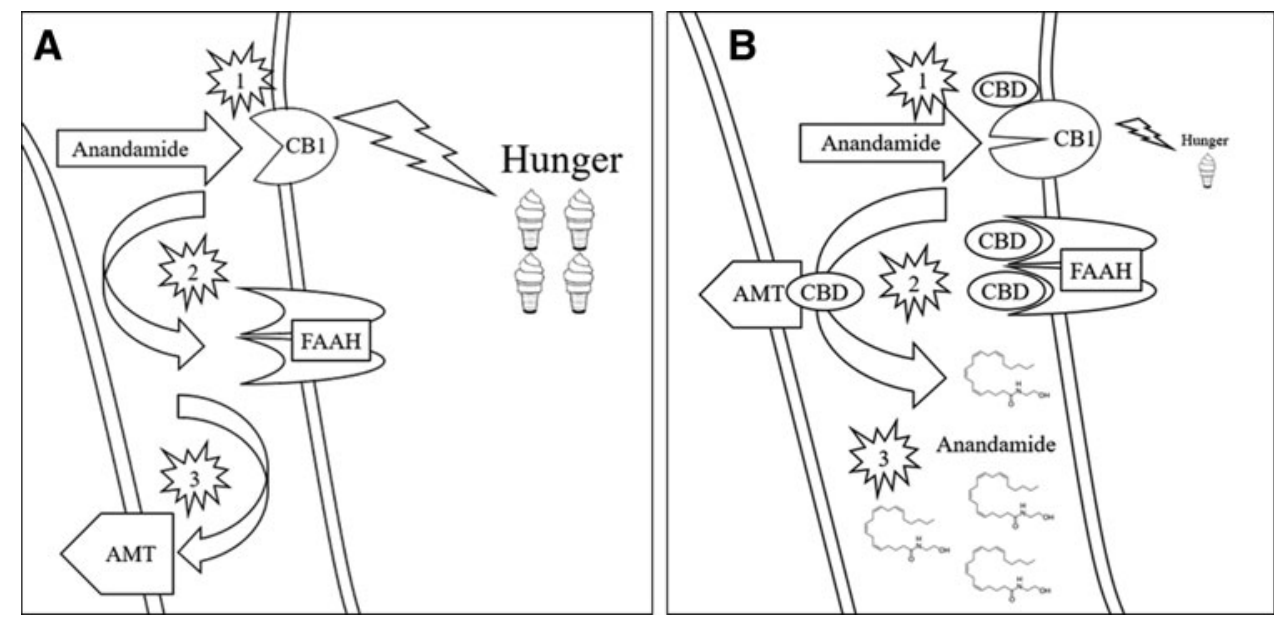

FIG. 1. Effects of CBD on hunger signaling. (A) Normal physiology. Step 1. Anandamide activates CB1 receptors driving hypothalamic circuits increasing hunger and food-seeking behavior. Step 2 . Anandamide is degraded by membrane enzyme FAAH. Step 3. Anandamide is cleared through AMT. (B) CBD effect. Step 1. $C B D$ is a negative allosteric modulator of $C B 1$ receptors and antagonizes activated receptors. Hunger is reduced. Step 2. CBD occupies binding sites of FAAH and AMT. Step 3. Circulating anandamide is increased. $A M T$, anandamide membrane transporter; $C B 1$, type 1 cannabinoid receptor; $C B D$, cannabidiol; FAAH, fatty acid amide hydrolase. 
model of hyperphagia, although there was no difference in food intake seen with CBD in fed or fasting state rats. ${ }^{41}$ In a recently published clinical trial investigating CBD for epilepsy, loss of appetite was reported in $28 \%$ of the treatment arm compared with $5 \%$ of the control $\mathrm{arm}^{42}$ and is currently being investigated in clinical trials as a treatment for hyperphagia in the Prader-Willi syndrome. CBD differs completely from rimonabant in chemical structure, pharmacological mechanism, and safety, ${ }^{43}$ therefore, CBD would not be expected to produce the serious neuropsychiatric effects seen with the rimonabant clinical experience. CBD may have relevance for the prevention of metabolic syndrome and insulin resistance through PPAR $\gamma$ agonism as reviewed by Kleiner. ${ }^{44}$ Finally, the possible $5 \mathrm{HT} 1 \mathrm{a}$ agonist effects may provide concurrent anxiolysis. ${ }^{45}$ A potential mechanism for the appetite suppressive effects of CBD is shown in Figure 1.

\section{Atherosclerosis}

CBD likely exerts an indirect effect on immune cell function by inhibiting the degradation of circulating endocannabinoids, thus increasing the availability to target type 2 CB receptors on neutrophils and macrophages in atherosclerotic plaques. CBD increases levels of the anti-inflammatory endocannabinoid anandamide but not proinflammatory $2-\mathrm{AG}^{46}{ }^{46}$ a finding that needs to be reproduced clinically. If present, this augmented "endocannabinoid tone" likely explains the finding of reduced IL- $1 \beta$, IL-2, IL-6, and TNF- $\alpha$ in experimental conditions. Until synthetic CB2 receptor agonists become available for clinical trials, the established safety profile and putative mechanism support the investigation of $\mathrm{CBD}$ as a therapeutic for atherosclerosis.

\section{Conclusion}

The rationale for $\mathrm{CBD}$ as a therapy for stroke prevention is shown in Figure 2. There is now a therapeutic opportunity to examine the effects of CBD in atherosclerosis and obesity. Synthetic cannabinoid agents although possibly more potent would not necessarily have the pleiotropic effects ascribed to CBD in the scientific literature. ${ }^{33}$ Although the role of endocannabinoids in

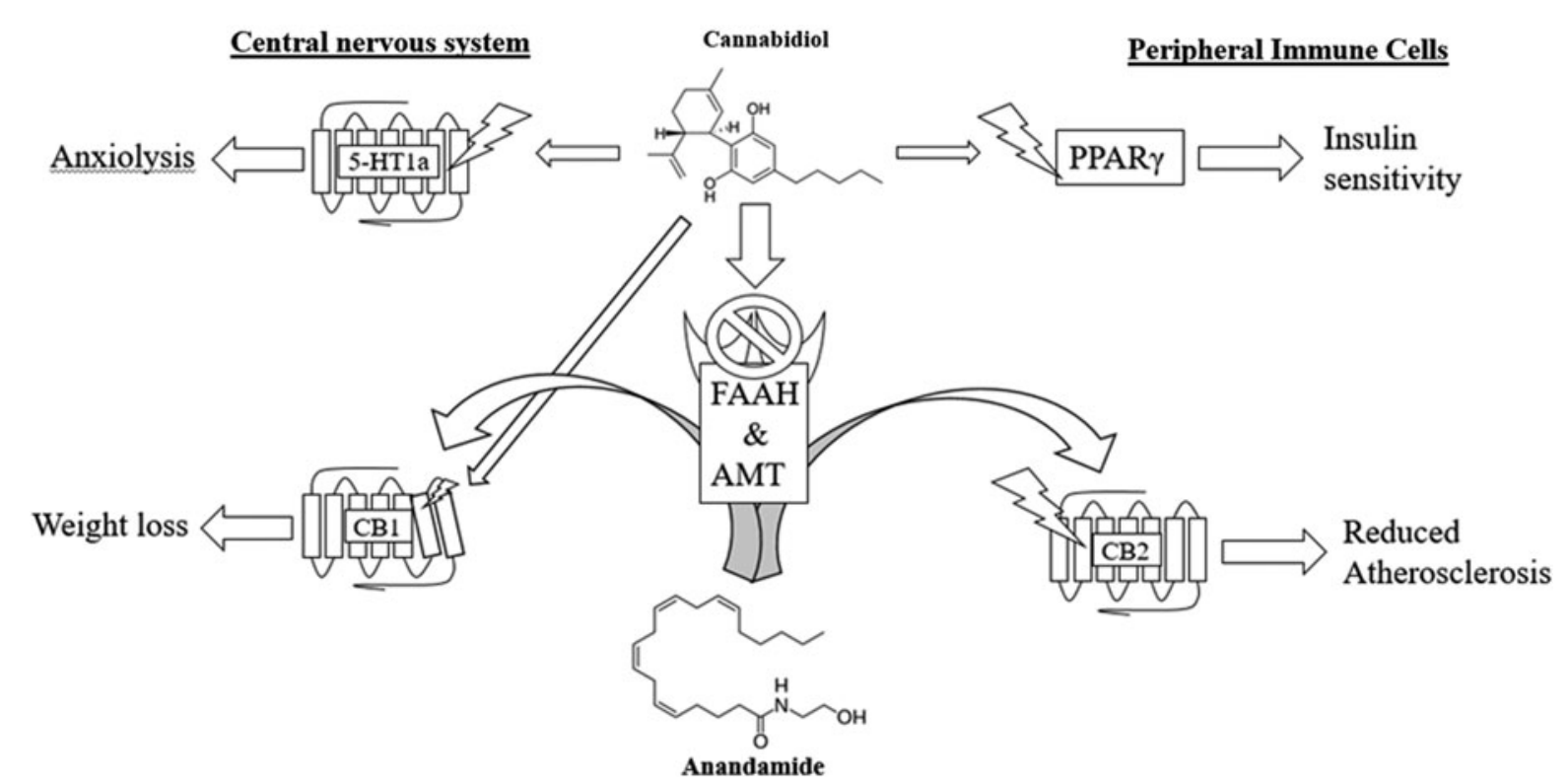

FIG. 2. Direct and indirect effects of $C B D$ on vascular and metabolic risk factors. $C B D$ (center) increases circulating anandamide by inhibiting the catabolic enzyme FAAH and transporter AMT. Central nervous system (left): CBD promotes anxiolysis through $5-\mathrm{HT} 1 \mathrm{a}$ activation. $\mathrm{CBD}$ reduces hunger signaling through negative modulation of activated CB1 receptors. Peripheral immune cells (right): CBD activates PPAR $\gamma$. Anandamide promotes $\mathrm{CB} 2$-mediated anti-inflammatory response, decreasing atherosclerosis on circulating neutrophils and lymphocytes. 5-HT1a, type 1a serotonin receptor; CB2, type 2 cannabinoid receptor; PPAR $\gamma$, peroxisome proliferator-activated receptor gamma. 
cerebrovascular disease should be studied, a current hypothesis that must be explored clinically is whether CBD can functionally antagonize central CB1 receptors while simultaneously increasing availability of circulating endocannabinoids (particularly anandamide) to promote an antiatherogenic vascular effect. Therefore, a clinical trial investigating CBD in vascular disease and obesity would serve an immediate public health need and is a necessary next step in advancing stroke prevention.

\section{Author Disclosure Statement \\ No competing financial interests exist.}

\section{References}

1. Committee on the Health Effects of Marijuana. An Evidence Review and Research Agenda; Board on Population Health and Public Health Practice; Health and Medicine Division; National Academies of Sciences. The Health Effects of Cannabis and Cannabinoids: The Current State of Evidence and Recommendations for Research. 2017. Available at www.nap.edu/24625 (accessed July 1, 2017).

2. Cota D, Marsicano G, Lutz B, et al. Endogenous cannabinoid system as a modulator of food intake. Int J Obesity. 2003;27:289-301.

3. Marsicano G, Lutz B. Expression of the cannabinoid receptor CB1 in distinct neuronal subpopulations in the adult mouse forebrain. Eur J Neurosci. 1999;11:4213-4225.

4. Felder CC, Joyce KE, Briley EM, et al. Comparison of the pharmacology and signal transduction of the human cannabinoid $\mathrm{CB} 1$ and $\mathrm{CB} 2$ receptors. Mol Pharmacol. 1995;48:443-450.

5. Jamshidi N, Taylor DA. Anandamide administration into the ventromedial hypothalamus stimulates appetite in rats. Br J Pharmacol. 2001;134: 1151-1154.

6. Kishimoto S, Gokoh M, Oka S, et al. 2-Arachidonoylglycerol induces the migration of HL-60 cells differentiated into macrophage-like cells and human peripheral blood monocytes through the cannabinoid CB2 receptor-dependent mechanism. J Biol Chem. 2003;278: 24469-24475.

7. Kishimoto S, Muramatsu M, Gokoh M, et al. Endogenous cannabinoid receptor ligand induces the migration of human natural killer cells. J Biochem. 2005;137:217-223.

8. Rayman N, Lam KH, Laman JD, et al. Distinct expression profiles of the peripheral cannabinoid receptor in lymphoid tissues depending on receptor activation status. J Immunol. 2004;172:2111-2117.

9. Oka S, Ikeda S, Kishimoto S, et al. 2-Arachidonoylglycerol, an endogenous cannabinoid receptor ligand, induces the migration of EoL-1 human eosinophilic leukemia cells and human peripheral blood eosinophils. J Leukoc Biol. 2004;76:1002-1009.

10. Gasperi V, Evangelista D, Chiurchiu V, et al. 2-Arachidonoylglycerol modulates human endothelial cell/leukocyte interactions by controlling selectin expression through CB1 and CB2 receptors. Int J Biochem Cell Biol. 2014;51:79-88.

11. Fiskerstrand $T, H^{\prime}$ Mida-Ben Brahim D, Johansson $S$, et al. Mutations in ABHD12 cause the neurodegenerative disease PHARC: an inborn error of endocannabinoid metabolism. Am J Hum Genet. 2010;87: 410-417.

12. Cencioni MT, Chiurchiu V, Catanzaro G, et al. Anandamide suppresses proliferation and cytokine release from primary human T-lymphocytes mainly via CB2 receptors. PLoS One. 2010;5:e8688.

13. Chiurchiu V, Cencioni MT, Bisicchia E, et al. Distinct modulation of human myeloid and plasmacytoid dendritic cells by anandamide in multiple sclerosis. Ann Neurol. 2013;73:626-636.

14. Sheng WS, Hu S, Min X, et al. Synthetic cannabinoid WIN55,212-2 inhibits generation of inflammatory mediators by IL-1beta-stimulated human astrocytes. Glia. 2005;49:211-219.
15. Rajesh M, Mukhopadhyay $P$, Batkai S, et al. CB2-receptor stimulation attenuates TNF-alpha-induced human endothelial cell activation, transendothelial migration of monocytes, and monocyte-endothelial adhesion. Am J Physiol Heart Circ Physiol. 2007;293:H2210-2218.

16. Ghosh S, Preet A, Groopman JE, et al. Cannabinoid receptor CB2 modulates the CXCL12/CXCR4-mediated chemotaxis of T lymphocytes. Mol Immunol. 2006;43:2169-2179.

17. Weis F, Beiras-Fernandez A, Sodian R, et al. Substantially altered expression pattern of cannabinoid receptor 2 and activated endocannabinoid system in patients with severe heart failure. J Mol Cell Cardiol. 2010;48:1187-1193.

18. Mohnle P, Schutz SV, Schmidt M, et al. MicroRNA-665 is involved in the regulation of the expression of the cardioprotective cannabinoid receptor CB2 in patients with severe heart failure. Biochem Biophys Res Commun. 2014;451:516-521.

19. Duerr GD, Heinemann JC, Dunkel S, et al. Myocardial hypertrophy is associated with inflammation and activation of endocannabinoid system in patients with aortic valve stenosis. Life Sci. 2013;92:976-983.

20. Engeli S, Bluher $M$, Jumpertz $R$, et al. Circulating anandamide and blood pressure in patients with obstructive sleep apnea. J Hypertens. 2012;30:2345-2351.

21. Ho WS, Hill MN, Miller GE, et al. Serum contents of endocannabinoids are correlated with blood pressure in depressed women. Lipids Health Dis. 2012;11:32.

22. Abdulnour J, Yasari S, Rabasa-Lhoret R, et al. Circulating endocannabinoids in insulin sensitive vs. insulin resistant obese postmenopausal women. A MONET group study. Obesity. 2014;22:211-216.

23. Quercioli A, Pataky Z, Vincenti G, et al. Elevated endocannabinoid plasma levels are associated with coronary circulatory dysfunction in obesity. Eur Heart J. 2011;32:1369-1378.

24. Bluher M, Engeli S, Kloting N, et al. Dysregulation of the peripheral and adipose tissue endocannabinoid system in human abdominal obesity. Diabetes. 2006:55:3053-3060.

25. Steffens S, Veillard NR, Arnaud C, et al. Low dose oral cannabinoid therapy reduces progression of atherosclerosis in mice. Nature. 2005;434: 782-786.

26. Gallily R, Breuer A, Mechoulam R. 2-Arachidonylglycerol, an endogenous cannabinoid, inhibits tumor necrosis factor-alpha production in murine macrophages, and in mice. Eur J Pharmacol. 2000;406:R5-R7.

27. Costa $B$, Trovato $A E$, Comelli $F$, et al. The non-psychoactive cannabis constituent cannabidiol is an orally effective therapeutic agent in rat chronic inflammatory and neuropathic pain. Eur J Pharmacol. 2007;556:75-83.

28. Gertsch J. Anti-inflammatory cannabinoids in diet: towards a better understanding of $\mathrm{CB}(2)$ receptor action? Commun Integr Biol. 2008;1:26-28.

29. Chiurchiu V, Lanuti M, Catanzaro G, et al. Detailed characterization of the endocannabinoid system in human macrophages and foam cells, and anti-inflammatory role of type-2 cannabinoid receptor. Atherosclerosis. 2014;233:55-63.

30. Montecucco F, Di Marzo V, da Silva RF, et al. The activation of the cannabinoid receptor type 2 reduces neutrophilic protease-mediated vulnerability in atherosclerotic plaques. Eur Heart J. 2012;33:846-856.

31. Bih $\mathrm{Cl}$, Chen T, Nunn AVW, et al. Molecular targets of cannabidiol in neurological disorders. Neurotherapeutics. 2015;12:699-730.

32. Laprairie RB, Bagher AM, Kelly ME, et al. Cannabidiol is a negative allosteric modulator of the cannabinoid CB1 receptor. Br J Pharmacol. 2015;172:4790-4805.

33. McPartland JM, Duncan M, Di Marzo V, et al. Are cannabidiol and Delta(9)tetrahydrocannabivarin negative modulators of the endocannabinoid system? A systematic review. Br J Pharmacol. 2015;172:737-753.

34. De Petrocellis L, Ligresti A, Moriello AS, et al. Effects of cannabinoids and cannabinoid-enriched Cannabis extracts on TRP channels and endocannabinoid metabolic enzymes. Br J Pharmacol. 2011;163: 1479-1494.

35. Bisogno T, Hanus $L$, De Petrocellis $L$, et al. Molecular targets for cannabidiol and its synthetic analogues: effect on vanilloid VR1 receptors and on the cellular uptake and enzymatic hydrolysis of anandamide. $\mathrm{Br} \mathrm{J}$ Pharmacol. 2001;134:845-852. 
36. Elmes MW, Kaczocha M, Berger WT, et al. Fatty acid-binding proteins (FABPs) are intracellular carriers for Delta9-tetrahydrocannabinol (THC) and cannabidiol (CBD). J Biol Chem. 2015;290:8711-8721.

37. Di Marzo V, Fontana $\mathrm{A}$, Cadas $\mathrm{H}$, et al. Formation and inactivation of endogenous cannabinoid anandamide in central neurons. Nature. 1994;372:686-691.

38. O'Sullivan SE, Sun Y, Bennett AJ, et al. Time-dependent vascular actions of cannabidiol in the rat aorta. Eur J Pharmacol. 2009;612:61-68.

39. Hind WH, England TJ, O'Sullivan SE. Cannabidiol protects an in vitro model of the blood-brain barrier from oxygen-glucose deprivation via PPARgamma and 5-HT1A receptors. Br J Pharmacol. 2016;173:815-825.

40. Pertwee RG. The diverse $C B 1$ and $C B 2$ receptor pharmacology of three plant cannabinoids: delta9-tetrahydrocannabinol, cannabidiol and delta9-tetrahydrocannabivarin. Br J Pharmacol. 2008;153:199-215.

41. Scopinho AA, Guimaraes FS, Correa FMA, et al. Cannabidiol inhibits the hyperphagia induced by cannabinoid-1 or serotonin-1A receptor agonists. Pharmacol Biochem Behav. 2011;98:268-272.

42. Devinsky O, Cross JH, Laux L, et al. Trial of cannabidiol for drugresistant seizures in the Dravet syndrome. N Engl J Med. 2017;376: 2011-2020.

43. Bergamaschi MM, Queiroz RH, Zuardi AW, et al. Safety and side effects of cannabidiol, a Cannabis sativa constituent. Curr Drug Saf. 2011;6: 237-249.

44. Kleiner D, Ditroi K. [The potential use of cannabidiol in the therapy of metabolic syndrome]. Orvosi hetilap. 2012;153:499-504.

45. Savitz J, Lucki I, Drevets WC. 5-HT1A receptor function in major depressive disorder. Prog Neurobiol. 2009;88:17-31.
46. Rimmerman N, Bradshaw HB, Kozela E, et al. Compartmentalization of endocannabinoids into lipid rafts in a microglial cell line devoid of caveolin-1. Br J Pharmacol. 2012;165:2436-2449.

Cite this article as: Scharf EL (2017) Translating endocannabinoid biology into clinical practice: Cannabidiol for stroke prevention, Cannabis and Cannabinoid Research 2:1, 259-264, DOI: 10.1089/ can.2017.0033.

\section{Publish in Cannabis and Cannabinoid Research}

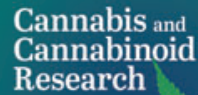

Research

- Immediate, unrestricted online access

- Rigorous peer review

- Compliance with open access mandates

- Authors retain copyright

- Highly indexed

- Targeted email marketing

liebertpub.com/can 